\title{
En búsqueda de mandatos infinitos: La elección y la reelección de gobernadores en las provincias de Chaco, Formosa y Misiones (1983-2015)
}

DOI: $10.15175 / 1984-2503-202012108$

\author{
Sergio David Valenzuela* \\ Marcos Walter Medina ${ }^{* *}$
}

\begin{abstract}
Resumen
La reforma política en las provincias argentinas, iniciada en 1987 con las modificaciones a las Constituciones provinciales, incorporó la posibilidad de reelección para los gobernadores. Este fenómeno, afectó al principio filosófico de origen republicano que ratifica las bondades alternancia en el poder, generando diferencias entre los distintos sistemas políticos provinciales de la región. En este marco, el presente trabajo tiene por objetivo comparar el impacto que tuvo la incorporación de los tipos de reelección para los cargos de gobernador y vicegobernador en los sistemas políticos provinciales en las provincias de Chaco, Formosa y Misiones. Los resultados dan cuenta de una serie de aspectos, sobre los cuales, es posible la reflexión teórica. Lo sobresaliente es que el número efectivo de personas que fueron electas, para el cargo de gobernador en las nueve elecciones que tuvieron las tres provincias, asciende a 16 , en un periodo en el que sin reelecciones debieran haber sido 27 y la competencia por el acceso a los cargos se torna bipartidista.
\end{abstract}

Palabras Claves: Reelección; ejecutivos subnacionales; sistemas políticos; Provincias del Nordeste; Argentina.

\footnotetext{
* Profesor de la Universidad Nacional del Nordeste (Argentina). Becario de Investigación Doctoral Cofinanciado Universidad Nacional del Nordeste - Consejo Nacional de Investigaciones Científicas y Técnicas. Profesor Asistente de Ciencias Políticas y Opinión Publica, Licenciatura en Publicidad, Facultad de Arte Diseño y Comunicación, Universidad de la Cuenca del Plata. Magister en Partidos Políticos (Centro de Estudios Avanzados -Facultad de Ciencias Sociales-Universidad Nacional de Córdoba). Candidato a Doctor en Historia por Facultad de Filosofía y Humanidades - Universidad Nacional de Córdoba. Miembro del Proyecto de Investigación Los cambios en los sistemas electorales y sus implicancias sobre los sistemas políticos provinciales. Análisis comparado de las provincias del nordeste argentino entre 1983 y 2013", Acreditado por la Secretaria General de Ciencia y Técnica de la Universidad Nacional del Nordeste. E-mail: sergiodavidvalenzuela@gmail.com. (1) https://orcid.org/0000-0003-1402-8584

** Abogado de la Universidad Nacional del Nordeste (Argentina). Magister en Estudios Sociales y Políticos Latinoamericanos Universidad Alfredo Hurtado (Chile). Profesor Adjunto a Cargo de la Catedra Fundamentos de Ciencias Políticas del Departamento de Comunicación Social, Facultad de Humanidades Sede Corrientes Capital, Universidad Nacional del Nordeste, Argentina. Director del Proyecto de Investigación Los cambios en los sistemas electorales y sus implicancias sobre los sistemas políticos provinciales. Análisis comparado de las provincias del nordeste argentino entre 1983 y 2013", Acreditado por la Secretaria General de Ciencia y Técnica de la Universidad Nacional del Nordeste. Profesor codirector de la beca de investigación y orientador de la investigación doctoral por mérito equivalente. E-mail: mrcsmedin@gmail.com. (10 https://orcid.org/00000002-8428-7074
} 
Em busca de mandatos infinitos. A eleição e reeleição de governadores nas províncias de Chaco, Formosa e Misiones (1983-2015)

\title{
Resumo
}

A reforma política nas províncias argentinas, iniciada em 1987 com as modificações nas Constituições provinciais, incorporou a possibilidade de reeleição para os governadores. Esse fenômeno afetou o princípio filosófico de origem republicana que ratifica os benefícios da alternância de poder, gerando diferenças entre os distintos sistemas políticos provinciais da região. Neste contexto, o presente trabalho tem como objetivo comparar o impacto causado pela incorporação da reeleição dos governadores e vice-governadores nos sistemas políticos das províncias de Chaco, Formosa e Misiones. Os resultados demonstram uma série de aspectos, sobre os quais é possível uma reflexão teórica. Chama a atenção o número efetivo de eleitos para o cargo de governador nas nove eleições ocorridas nas três províncias, somando um total de 16 pessoas. Nesse mesmo período, sem reeleições, normalmente seriam 27 pessoas. Dessa forma, a disputa pelo acesso aos cargos tornou-se bipartidária.

Palavras-chave: Reeleição; executivos subnacionais; sistemas políticos; Províncias do Nordeste; Argentina.

In search of infinite mandates. The election and re-election of governors in the provinces of Chaco, Formosa, and Misiones (1983-2015)

\begin{abstract}
Political reform in Argentina's provinces, which began in 1987 with the modifications made to the provincial constitutions, incorporated the possibility of governors' re-election. Such a phenomenon affected the main philosophy of Republican origin that upheld the benefits of rotations in power, generating differences between the different provincial political systems in the region. In such a framework, the work aims to compare the impact of the incorporation of the types of re-election for the posts of governor and vice-governor in the provincial political systems of Chaco, Formosa, and Misiones. The results reveal a series of findings to have prompted theoretical reflections. The main finding is that the effective number of people elected to the post of governor in the nine elections held in the three provinces rose to 16 in a period which would have had 27 without re-elections, and that the competition turned bipartisan in accessing the posts.
\end{abstract}

Keywords: Re-election; sub-national executives; political systems; northeastern provinces; Argentina.

En quête de mandats infinis. L'élection et la réélection des gouverneurs des provinces de Chaco, Formosa et Misiones (1983-2015)

\section{Résumé}

La réforme politique mise en œuvre dans les provinces argentines à partir de 1987 par le biais de modifications apportées aux constitutions provinciales a intégré la possibilité de réélection des gouverneurs. Ce phénomène a affecté le principe philosophique et républicain d'alternance du pouvoir, provoquant ainsi des disparités entre les différents systèmes politiques provinciaux du pays. Le présent article a ainsi pour but de comparer l'impact de l'intégration de la réélection des gouverneurs et vice-gouverneurs dans les systèmes politiques des provinces de Chaco, Formosa et Misiones. Les résultats obtenus rendent compte d'un ensemble d'éléments à propos desquels il s'avère possible de mener une réflexion théorique. Ce qui en ressort est que le nombre effectif de personnes ayant été élues au poste de gouverneur lors des neuf élections organisées dans les trois provinces a été de seize, et ce au cours d'une période qui, sans réélection, aurait permis l'accession audit poste de vingt-sept candidats, et que la concurrence électorale y afférente est devenue bipartite. 
Mots-clés : Réélection ; exécutifs provinciaux; systèmes politiques; Provinces du Nord-Est ; Argentine.

\section{寻求无限的任期一阿根廷查科，福尔摩沙和米西奥内斯省的省长选举和连任（1983-2015）}

\section{摘要 :}

阿根廷各省的政治改革始于1987年, 当时各省都对宪法进行了修改, 解除了对省长连选连任的限制 。这种现象影响了共和派的权力定期交替的政治原则。该原则认可了权力交替产生的利益, 从而在 该地区不同的省级政治体系之间产生了分歧。在这种情况下, 本文旨在比较连任型选举对查科, 福 尔摩沙和米西奥内斯省的省级政治体系的影响。根据统计, 在三个省的九次选举中, 新省长的当选 人数为 16 人, 而在没有连选连任的情况下, 他们应该是 27 人, 并且进入了两党制竞争。我们分析了 1987年修宪后，允许连任所产生的政治竞争和政治生态变化，并作了理论上的反思。

关键字：连任；地方行政人员；政治制度；东北省；阿根廷

\section{Introducción 1}

Estudiar el poder ejecutivo en Argentina, ya sea en el orden nacional como subnacional, implica considerar que el sistema de gobierno adoptado por el país es presidencialista. A su vez estudiar el poder ejecutivo, implica entender que en Argentina es monista y monocrático, esto es, una sola persona a cargo de este poder de gobierno (SÁlZ ARNAIZ, 1997). Desde esta perspectiva, es importante avanzar en los estudios que den cuenta de las problemáticas que se generan en torno al poder, cuando este debe ser ejercido por una sola persona por un periodo fijo y prolongado en el tiempo.

Los marcos filosóficos del republicanismo, sostienen el principio de la alternancia en el poder. Este principio, consagrado como constitucional en la actualidad de muchos países, y en este escenario se abre el debate sobre la perpetuación en los cargos electivos en los distintos niveles de gobierno de cada país (GARGARELLA, 1995; SERRAFERO, 2011).

Thibaut (1993), señala que los países de América Latina, donde una persona acumula los poderes de jefe de Estado y de jefe de gobierno, históricamente la tendencia fue la prohibición de la reelección inmediata de los ejecutivos. Sobre esto, Linz (1997) revela que el origen de la prohibición, se encuentra en el Tratado General de la Paz y la Amistad firmado en Washington por países centroamericanos en 1927, y que mantuvo la prohibición

\footnotetext{
1 Una versión preliminar del trabajo fue presentada en el XV Congreso Nacional de Derecho Político desarrollado en la Ciudad de Córdoba entre el 3 y el 5 de junio de 2019, organizado por la Asociación Nacional de Profesores de Derecho Político y la Facultad de Derecho de la Universidad Nacional de Córdoba. El trabajo se tituló "El camino a la perpetuación. Procesos de reelección de gobernadores en las provincias de Chaco, Formosa y Misiones (1983-2015)" y los trabajos del evento no han sido publicados.
} 
hasta finales del siglo XX. Esta discusión se amplía, en situaciones como la de Argentina, ya que además el país organiza el territorio de modo federal. En este escenario, cada unidad territorial subnacional o subestatal, tiene sus propios gobiernos, sus propias constituciones y por ende sus propias reglas de juego político.

Dichas reglas de juego, se materializan en los sistemas electorales. Estos últimos, por definición son los mecanismos que traducen los votos en cargos (MOLINELLI, 1998; NOHLEN, 1999). Respecto de los componentes de los sistemas electorales los autores citados, describen a los siguientes: la selección de candidatos, la magnitud del distrito, la conformación de listas, la fórmula electoral, el umbral o piso electoral. El trabajo de Nohlen (1999), señala que los sistemas electorales avanzan y cambian más rápidamente, que las investigaciones en Ciencia Política. Molinelli (1998), por su parte, plantea que ningún sistema electoral es perfecto, todos tienen puntos fuertes y grandes falencias, y que el contexto institucional tiene influencias sobre sus relaciones con los votantes. Esto último se ejemplifica, notando que en un país federal como Argentina, por lo general las elecciones presidenciales tienen un efecto de arrastre sobre las elecciones a gobernadores.

En cuanto a las investigaciones sobre los sistemas electorales, existen grandes enfoques y vertientes, que en general tienen que ver con distintos modos de observar y analizar el funcionamiento de los mismos. Desde el enfoque en el que se aborda la temática, resulta necesario expresar que los representantes de las líneas de investigación que analizan los efectos sociológicos de los sistemas electorales (APTER; ECKSTEIN; HECKSCHER, 1963; LIPSET; ROKKAN, 1967; NOHLEN, 1981, 1994; ROKKAN, 1970; entre otros), niegan la existencia de una relación de carácter mecánica entre los sistemas electorales y los sistemas de partidos. Los referentes de ésta corriente, sostienen que los efectos de las leyes electorales están condicionados por las estructuras sociopolíticas específicas de cada territorio, a partir de lo cual, un sistema electoral no funciona de la misma manera en diferentes sociedades, circunscripciones o momentos diferentes.

Una de las leyes electorales más discutidas por los analistas, para los casos en los sistemas políticos que adoptan el presidencialismo como forma de gobierno, es la reelección de los ejecutivos, tanto a nivel nacional como subnacional, ya que como se señaló al principio posibilita o genera condiciones que promueven la perpetuación de personas frente a cargos unipersonales. La clasificación propuesta por Serrafero (2011), si bien esta realizada en base a la reelección presidencial ha sido muy difundida y utilizada para los demás niveles de gobierno. El autor señalado remite a dos extremos, uno en el que se ubica la prohibición absoluta de la reelección y otro donde se ubica la reelección sin 
límites o indefinida; y en el medio del extremo un continuum donde se ubican la reelección mediata (luego de un intervalo de tiempo) y la reelección inmediata, en ambos casos pueden ser cerradas (por única vez) o abiertas (se puede volver a postular para acceder al cargo luego de un lapso de tiempo establecido por la ley electoral).

En función de lo señalado, es posible considerar que la perpetuación en los cargos ejecutivo provinciales obedece a cambios políticos que en Argentina a nivel subnacional van en dos sentidos. Por un lado, la incorporación de la posibilidad de reelección, mediante reformas en las constituciones provinciales. Por otro lado, los comportamientos políticos de los líderes frente a la posibilidad de ser reelegidos en sus cargos, cualquiera sea el nivel de gobierno que ocupe y el poder del Estado en el que se desempeñe.

En relación con estos elementos, existe producción que avanzó en la interpretación de los cambios en las reglas de juego. En primer lugar, De Riz (1992) describe y explica el debate que se generó en torno de la reforma electoral en las provincias argentinas. Respecto de los modos elección del poder ejecutivo, la autora señala lo controversia que se presentó en relación a la adopción del doble voto simultáneo o ley de lemas en las diferentes jurisdicciones. En términos generales, este mecanismo fue aprobado en las distintas provincias que lo adoptaron por diferencias mínimas, lo cual reflejaba el alto grado de disenso que esta generaba al interior de la clase política.

En segundo lugar, Corbacho (1998), analiza los cambios en las constituciones que afectan los modelos de decisión en las provincias argentinas. El autor explicando los cambios que se desarrollaron en las provincias, con respecto a la reelección de los gobernadores, señala que las provincias en líneas generales en la transición democrática presentaban dos posiciones coincidentes con los modelos propuestos Lijphart (1994). De esta manera algunas provincias, tenían una posición más cercana a las democracias consensuales y se oponían a la incorporación de la reelección inmediata de los gobernadores; mientras que otras provincias más cercanas a las democracias mayoritarias, impulsaban la adopción de la posibilidad de reelección inmediata para los ejecutivos provinciales. Con el correr del tiempo y a partir de las reformas las segunda posición comenzaba a ser tomada por la mayor parte de los distritos.

En tercer lugar, dentro de la variedad de trabajos que existen en torno a la temática de la reforma de los sistemas electorales en las provincias argentinas, dentro de los que interesa rescatar la mirada analítica de Ratto (2013), quien estudia las estructuras de competencia partidaria en las provincias argentinas a partir de las reformas electorales efectuadas en los distritos entre 1983 y 2005. La autora, a partir del análisis de los factores 
que subyacen a las reformas subnacionales, concluye señalando que la competencia partidaria efectiva se asocia a tres elementos: a) en relación a la fragmentación partidaria, la competencia se con la cantidad efectiva de cargos a elegir; b) la fortalezade la Unión Cívica Radical y el Partido Justicialista, dado que estas fuerzas tradicionales durante las reformas contaban las mayorías legislativas; y c) el factor que refleja las reformas en términos de la autora es el sistema electoral y no la cantidad de cargos a elegir, dado que la permisividad de las normas imprimió la estructura de la competencia electoral.

En función de los cambios en las reglas de juego mencionados, considerando las provincias de Chaco, Formosa y Misiones, entre 1983 y 2015; este trabajo intenta responder a una serie de interrogantes asociados a la perpetuación de las personas frente al cargo de gobernador en estas provincias. La gran pregunta es ¿por qué algunos gobernadores consiguieron con el paso del tiempo obtener reelección indefinida y otros no? ¿A qué se debe que en cada una de estas provincias que integran una misma región geográfica en el país, existan reglas de juego político diferentes? $Y$ finalmente considerando que las jurisdicciones cuentan con sistemas electorales y posibilidades constitucionales de reelección para el gobernador diferencias ¿qué efectos tienen estas diferencias sobre los regímenes políticos provinciales?

A partir de los interrogantes señalados a modo de hipótesis, es posible afirmar que en las provincias analizadas, no se cumple el argumento que sostiene que las reelecciones son instituciones forzadas por los gobernadores. La prueba contra fáctica que derriba este argumento, es el hecho que en dos de las tres provincia analizadas, los gobernadores que propician la modificación constitucional para incorporar la posibilidad de ser reelegidos inmediatamente, no se postulan para acceder nuevamente al cargo. Sin embargo, si se cumplen los postulados de la corriente e investigación sociológica sobre los efectos de los sistemas electorales, que sostienen que las reglas electorales dependen de las estructuras sociopolíticas específicas. Esto sugiere, que a pesar de la cercanía y los puntos en común que tienen las provincias estudiadas, sus culturas políticas resultan diferentes y esto se refleja en los resultados electorales a lo largo del tiempo.

En este marco, este trabajo tiene por objetivo analizar comparativamente el impacto de los cambios de las reglas de juego político en torno a los cargos del poder ejecutivo provincial entre 1983 y 2015, dentro de los cuales se destaca la incorporación de distintos tipos de reelección para los cargos de gobernador y vicegobernador en los regímenes políticos provinciales de Chaco, Formosa y Misiones. 
En función del objetivo señalado y de la hipótesis planteada, el desarrollo del trabajo avanzara secundariamente sobre el sistema de partidos imperante en cada provincia. Dado que en ciencia política, existen diversas clasificaciones es conveniente aclarar que en este trabajo, se considerará la propuesta de Sartori (1980) que se puede entender como una (instancia) investigación/interpretación superadora de lo escrito por Maurice Duverger (1957), ya que es una tipología no solo atiende al número de partidos existentes en un sistema político, sino también a los aspectos ideológicos.

En relación con el estado del arte, el desarrollo de la política subnacional dentro del campo de la ciencia política en Argentina, cuenta con una serie de trabajos que vienen hace ya más de dos décadas explicando el funcionamiento de los regímenes provinciales y las reformas políticas que se desarrollaron (TULA, 1995, 2001; GERVASONI, 2009). Dentro de la gama de trabajos que ofrece la política subnacional, a continuación se mencionan algunos que resultan útiles en función de los casos considerados en este trabajo.

Almaraz (2010), siguiendo el trabajo de Corbacho (1998), explica los procesos de reformas constitucionales por los que se fue incorporando en cada una de las provincias la posibilidad de reelegir el gobernador. A partir de estas reformas, esta investigadora da cuenta de cómo la ambición por el poder de los gobernadores en ejercicio, generó una ola de modificaciones en la gran mayoría de las constituciones provinciales en pos de incorporar la posibilidad de reelección. La autora concluye señalando que la incorporación de la posibilidad de ser reelecto para los gobernadores, dependió en gran medida de los contingentes legislativos con los que contaban los gobernadores o los partidos. No obstante, a pesar de que las afirmaciones planteadas de esta autora, en la comparación a nivel regional con menor generalidad, estas explicaciones son discutibles.

Jolías y Reina (2011) analizando las duraciones reales de los mandatos de los gobernadores de Argentina y Brasil, avanzan sobre la hipótesis de que las reelecciones son instituciones forzadas por los gobernadores. Esta hipótesis es aplicada válidamente para explicar el comportamiento de algunos gobernadores argentinos, que fueron introduciendo reformas a las constituciones provinciales de modo que las reglas de juego político siempre le resulten favorables.

Ortiz de Rozas (2011) realiza unos señalamientos teóricos importantes a la hora de realizar el análisis de los regímenes políticos subnacionales. La autora señala que 
a la capacidad de conformar fuerzas políticas que se perpetúan en el poder provincial y son electos con porcentajes muy altos (ORTIZ DE ROZAS, 2011, p. 362).

La autora concluye que las relaciones clientelares se construyen a partir del intercambio de bienes materiales y simbólicos, relaciones que luego se transforman en lealtades que legitiman las fortaleces a las que llegan los oficialismos que terminan resultando invencibles electoralmente.

Cardarello (2012), señala que la ambición por la reelección de los gobernadores materializa el anhelo de inmortalidad dentro del sistema político local. Este autor, describe los tipos de reelección vigentes en cada provincia y demuestra cómo impactó la posibilidad de reelección inmediata para los gobernadores sobre los sistemas provinciales. El análisis propuesto por el autor concluye argumentando que, en cada una de las provincias argentinas las reglas de juego político son diferentes y por lo tanto se generan estructuras de competencia partidarias diferenciadas en cada una de las provincias.

Garay (2017) estudia la alternancia en los poderes ejecutivos subnacionales de Argentina en 1983 y 2011, partiendo de la hipótesis de un bipartidismo extendido observable en los procesos de competencia electoral a nivel de las provincias, brinda una apertura a nuevos surcos de investigación sobre los procesos de cambio en las preferencias del electorado y como las modificaciones que se van incorporando en las reglas de juego, de la competencia electoral se configuran de diferente manera a nivel territorial. En su conclusión sostiene que es posible distinguir patrones comunes entre las provincias con y sin alternancia; y al grado de implicancia de los sistemas electorales provinciales y las modificaciones en los sistemas electorales tienen consecuencias importantes en la competencia política provincial las que, a su vez, repercuten sobre el formato del sistema de partido subnacional.

Además, de estos trabajos vinculados a la política subnacional de argentina en general, en la producción académica de la Ciencia Política, existen trabajos que se refieren puntualmente a las provincias estudiadas.

Beck (2012) estudiando el sistema político de la Provincia de Formosa, señala que se trata de un caso donde el Partido Justicialista construye hegemonía a partir del retorno de la democracia. El autor señala que:

desde el retorno de la democracia en 1983 hasta el presente en Formosa todas las elecciones a gobernador fueron ganadas por el justicialismo con porcentajes que se ubicaron entre el 42,8\% en 1983 hasta el $75 \%$ en 2011. Una revisión de los hombres que alternaron diversos cargos gubernamentales durante estas últimas décadas pone en evidencia la endogamia del partido oficialista (BECK, 2012, p. 42). 
A partir de ello, el autor analiza las redes clientelares construidas en la provincia en estudio, y señalando que el clientelismo es una deformación de la democracia concluye argumentado que entender a los actores políticos como personas necesitadas es diferente de concebirlas como poseedoras de preferencias, intereses y valores; y la persona necesitada no es dueña de sus preferencias, sino prisionera de sus necesidades, por lo cual los votos terminan siendo intercambiados por dinero como mercancías.

Quilici (2015) realizando un estudio sobre la coordinación entre niveles electorales en Estados multiniveles se dedica a comparar las jurisdicciones de Corrientes y Misiones. Dado que en este trabajo no se incluye a Corrientes, se hará una referencia a las referencias sobre Misiones. El autor argumenta que desde el retorno a la democracia hacia el presente existieron profundos cambios en el sistema de partidos y señala que:

\begin{abstract}
entre 1983 y 1999, la estabilidad del sistema político fue una de sus principales características, la Unión Cívica Radical y el Partido Justicialista obtuvieron siempre más del $90 \%$ de los votos en todas las categorías en cuestión [...] En la elección de 2011 , la UCR obtuvo el $6 \%$ de los votos y la coalición donde participó el justicialismo apenas logró el 3\% de los votos (QUILICI, 2015, p. 102).
\end{abstract}

El autor en la descripción de los procesos electorales desarrollados, da cuenta del surgimiento del Frente Renovador para la Concordia Social, un partido provincial en el que convergen liderazgos del justicialismo y el radicalismo local, por lo cual a partir de las disputas al interior de estos grandes partidos nacionales, el nacimiento de este frente provoca una fuerte ruptura en el escenario provincial. En este marco, el autor concluye señalando que en este caso, que el sistema de partidos que hasta 1999 estuvo completamente nacionalizado, paulatinamente comienza a regionalizarse, fenómeno que llega a su punto cumbre en las elecciones del 2007 cuando el Frente renovador consigue la victoria electoral con más del $65 \%$ de los votos.

Martin y Carlino (2018), analizando los cambios en las reglas de juego político en el régimen político de la provincia de Misiones, desde una visión histórica en clave política. Las autoras analizan las leyes sustantivas que se sancionaron en los últimos treinta años en la provincia y aquellas leyes y reformas parciales que afectaron al sistema electoral. Concluyen argumentando que las reformas electorales lejos de ser un problema técnico, son complejas y multidimensionales, y se enmarcan en una cultura abonada de ilegalidad y anomia o se desapego de la ley, llegando a tolerar leyes inconstitucionales por largo tiempo y en la provincia que analizan en el trabajo estas cuestiones tienen su origen en la provincialización del territorio nacional a mediados del siglo XX. 


\section{Metodología}

En cuanto a los aspectos metodológicos, resulta importante destacar que el universo de estudio está comprendido por tres provincias que integran la región Nordeste de la Argentina, Chaco, Formosa y Misiones. Se excluyó a la provincia de Corrientes, dado que las intervenciones federales dieron como resultado, un número de interventores y personas a cargo del poder ejecutivo mayor al de los gobernadores electos democráticamente en el periodo.

El recorte temporal inicia en 1983, dado que se llevan adelante las primeras elecciones que marcan el retorno a la Democracia en la República Argentina; y como cierre el año 2015, año en que tres de las cuatro provincias tuvieron elecciones a gobernador. En éste periodo, en las provincias mencionadas, se llevaron adelante nueve elecciones para los cargos de gobernador y vicegobernador.

Se utilizó el método comparativo, dado que permite trabajar con semejanzas y diferencias, para llegar a establecer casos similares y casos diferentes (SARTORI; MORLINO, 1994; PASQUINO, 2004; PÉREZ LIÑÁN, 2008). El paralelismo se establece a partir del número efectivo de personas que ejercieron el cargo de gobernador, habiendo sido electas. Además, fue necesario mencionar, la cantidad de reformas constitucionales realizadas, ya que tuvieron incidencia sobre el número efectivo de gobernadores.

En cuanto al método seleccionado, conviene señalar que es amplio y ofrece muchas posibilidades de comparación, aquí se avanzará considerando la comparación de casos similares y casos diferentes. Por esta razón, a partir de las provincias analizadas se buscará establecer en cuáles son similares entre si y cuáles diferentes.

En relación con los procedimientos, se llevaron adelante los siguientes: 1) identificación de las cambios de las reglas de juego político asociadas a la posibilidad de permanencia de las personas frente los cargos ejecutivos y al sistema electoral; 2) determinación del número efectivo de personas electas para los cargos de gobernador y vicegobernadores en cada una de las provincias en estudios; 3 ) tabulación de los resultados electorales para el poder ejecutivo de cada provincia teniendo en cuenta candidatos, partidos políticos y porcentajes de votos obtenidos; y 4) análisis e interpretación de los resultados a partir de los encuadres teóricos.

En cuanto al diseño metodológico, la variable independiente es la posibilidad reelección de los gobernadores, las variables independientes son el sistema de partidos y el sistema electoral, y los indicadores están constituidos por los resultados electorales y las reformas políticas y constitucionales que afectan al poder ejecutivo. 


\section{Resultados y discusión}

Los resultados dan cuenta de una serie de cambios de reglas de juego político, sobre los cuales, resulta valiosa la reflexión teórica. En función de ello, resulta necesaria la descripción de los procesos de reforma que afectaron al poder ejecutivo en las provincias de Chaco, Formosa y Misiones.

La provincia del Chaco, no tuvo números procesos de reforma, sino que las reformas que permanecieron en el tiempo se dieron en un mismo proceso, no obstante conviene detallar los hechos en su contexto. En un marco de reforma electoral en el que muchas provincias adoptaron la ley de lemas o el mecanismo de doble simultaneo en la segunda mitad de 1990, la provincia adopto la ley de lemas para la elección a gobernador; sin embargo en la primera mitad de 1991 fue rápidamente abolida (DE RIZ, 1992). Luego de esto, en un proceso de reforma de la Constitución Provincial, se realizaron varios cambios a saber: a) incorporación de la reelección inmediata y abierta para el ejecutivo provincial; b) cambio de forma de elección del gobernador, a través de la que se incorpora la doble vuelta si el candidato ganador no supera el $45 \%$ de los sufragios válidos o supera el $40 \%$ con 10 puntos de ventaja sobre el segundo; y c) los intendentes dejan de ser electos por cuerpos colegiados y pasan a ser electos directamente por el voto popular (MICOZZI, 2001).

La provincia de Formosa, en el lapso de tiempo estudiado atraviesa por una serie de procesos de reformas políticas que modelaron distintas maneras el funcionamiento de la política provincial. De esta manera, se destacan los siguientes fenómenos que afectaron el marco legal institucional con incidencia en la política de la provincia: a) la incorporación de la ley de lemas en 1987 para todos los cargos electivos en la provincia (DE RIZ, 1992; BECK, 2012), derogada parcialmente en el 2011, cuando se la dejó sin efecto para la elección de los cargos de gobernador y vicegobernador; b) reforma constitucional en 1991, en la que se incorporó la posibilidad de reelección inmediata para los cargos de gobernador y vicegobernador, donde además se aclaró que "si fueran reelectos o se sucedieran recíprocamente, no podrían luego ser elegidos para ninguno de ambos cargos, sino con el intervalo de un período" (BECK, 2012, p. 41); y c) reforma constitucional en el 2003 donde se incorpora la relección indefinida para los cargos electivos provinciales (BECK, 2012).

La provincia de Misiones por su parte, fue un escenario donde se desarrollaron fenómenos de reforma política similares en alguna medida a los que sucedieron en Formosa, pero con importantes matices diferenciadores. Martín y Carlino (2018), se refieren a la secuencia de numerosos eventos que afectaron el sistema institucional de la provincia, 
dentro de los cuales a los fines de este trabajo interesan los siguientes: a) la incorporación de la reelección inmediata para los cargos de gobernador y vicegobernador en 1988 y ratificada en 1989 de acuerdo al requerimiento de la propia constitución; b) la introducción de la ley de lemas en las elecciones provinciales en 1990, en principio utilizada para todos los cargos provinciales en las elecciones provinciales de 1991 y 1995; c) dicho mecanismo electoral en 1996 fue derogado para las elecciones de los cargos provinciales y supeditado al ámbito municipal, donde también resulto suspendida entre finales del 2003 y principios del 2006, al presente dicha ley se encuentra vigente para las elecciones de cargos municipales. Al relato de estas autoras, considerando que los objetivos de este trabajo focalizan las reformas vinculadas a la reelección, conviene agregar que en el año 2006 el gobernador impulsó la incorporación de la cláusula de posibilidad de reelección indefinida, sin embargo, la oposición triunfo en la elección de constituyentes obteniendo 20 de los 35 convencionales. A partir de ello, la convención se reunió bajo la presidencia del obispo Joaquín Piña líder del Frente Unidos por la Dignidad, y no aprobó la modificación, ratificando la modalidad de reelección inmediata por un periodo o la posibilidad de sucesión entre gobernador vice y sin a derecho a un nuevo mandato.

A partir de las modificaciones implementadas desde el retorno a la democracia al presente, es posible a partir de los guarismos generar discusiones y reflexiones teóricas. En relación al número efectivo de gobernadores se puede realizar el siguiente guarismo, considerando 9 elecciones sucesivas en tres jurisdicciones sino hubiera reelección serían electas para ocupar el cargo 27 personas, contra fácticamente si hubiera reelección indefinida y fuera plenamente exitosa serian electas sólo 3 personas para la cartera. Sin embargo, la realidad da cuenta de que fueron electas 16 personas para el cargo, por lo cual puede deducir que el efecto de la reelección fue diferenciado en cada distrito.

Estas cuestiones dan prueba de que el funcionamiento de los sistemas electorales depende de estructuras sociopolíticas particulares, como lo argumentan los trabajos de Apter, Eckstein y Heckscher (1963), y Lipset y Rokkan (1967). Teniendo en consideración éstos elementos, las reglas del juego político no devienen sólo en letra muerta cimentadas en legislación, sino que además dan cuenta de particularidades de la cultura política local.

Los procesos a escala local dan cuenta de diferentes comportamientos no solo frente a la reelección sino dentro de los sistemas políticos provinciales. Si bien, en este trabajo la metodología utilizada no alcanza para explicar los procesos que ocurren al interior de la cultura política de cada una de las provincias, se pueden señalar algunas diferencias. La búsqueda paulatina de la reelección indefinida en Formosa fue exitosa y como lo señala la 
propuesta citada de Beck (2012), la sociedad de pareciera aceptar y convalidar la maquinaria electoral aceitada que logra funcionamiento pleno y facilita la perpetuación del gobernador.

En Misiones, sin embargo, las mismas ambiciones de la clase política y del líder partidario no tuvieron el mismo respaldo de la sociedad, que no aportó con sus votos el aval a la reforma política que impulsaba la incorporación de la reelección indefinida. A pesar de los arreglos institucionales, la sociedad no permitió que la concentración del poder político terminara recayendo en manos del gobernador. No obstante, las evidencias demuestran que el partido de gobierno si pudo consolidarse.

En el Chaco, las cláusulas de reelección indefinida para el gobernador tampoco se llegaron a incluir, y el trasfondo políticos tan diferenciado de las otras jurisdicciones, se evidencia en el hecho de que se la ley de lemas no llegó a aplicarse. Además, para elección del gobernador, se incorporó la doble vuelta electoral, con lo cual más allá del funcionamiento empírico de la norma, podría pensar que dificulta la llegada y permanencia de los gobernadores en el poder.

Los resultados electorales y las diferencias porcentuales entre el ganador y el segundo candidato más votado de acuerdo a sus filiaciones partidarias, permiten observar el modo en que los diferentes cambios en las reglas de juego, afectaron a los sistemas partidarios locales. Las tablas que se presentan a continuación, permiten ilustrar a través de los resultados electorales cómo evolucionó en el período en estudio, el sistema de partidos al interior de cada uno de los distritos.

Tabla 1: Porcentajes de votos obtenidos por el ganador y el segundo candidato más votado, en elecciones para gobernador de Formosa 1983-2015.

\begin{tabular}{|c|c|c|c|c|c|c|c|c|c|}
\hline Elecciones & 1983 & 1987 & 1991 & 1995 & 1999 & 2003 & 2007 & 2011 & 2015 \\
\hline $\begin{array}{l}\% \text { del } \\
\text { Ganador }\end{array}$ & 45,12 & 52,63 & 42,46 & 50.8 & 63,36 & 53.36 & 46.86 & 66.59 & 52.73 \\
\hline Partido $^{2}$ & PJ & PJ & PJ & PJ & PJ & PJ & PJ & PJ & PJ \\
\hline Candidato & $\begin{array}{c}\text { F. } \\
\text { Bogado }\end{array}$ & V. Joga & V. Joga & $\begin{array}{c}\mathrm{G} . \\
\text { Insfran }\end{array}$ & $\begin{array}{c}\mathrm{G} . \\
\text { Insfran }\end{array}$ & G. Insfran & G. Insfran & G. Insfran & G. Insfran \\
\hline $\begin{array}{l}\text { \% del } \\
\text { segundo }\end{array}$ & 37,20 & 46,50 & 33,64 & 39,12 & 26,06 & 22.07 & 17,9 & 24,06 & 25,97 \\
\hline Partido & UCR & UCR & $\begin{array}{l}\text { Frente } \\
\text { Amplio }\end{array}$ & UCR & UCR & $\begin{array}{c}\text { Frente de } \\
\text { Todos }\end{array}$ & UCR & UCR & UCR \\
\hline Candidato & $\begin{array}{c}\mathrm{R} . \\
\text { Maglietti }\end{array}$ & $\begin{array}{c}\text { R. } \\
\text { Maglietti }\end{array}$ & $\begin{array}{c}\text { R. } \\
\text { Maglietti }\end{array}$ & $\begin{array}{c}\mathrm{R} . \\
\text { Maglietti }\end{array}$ & V. Joga & $\begin{array}{c}\text { G. } \\
\text { Hernández }\end{array}$ & $\begin{array}{c}\text { L. } \\
\text { Naidenoff }\end{array}$ & $\begin{array}{c}\text { L. } \\
\text { Naidenoff }\end{array}$ & $\begin{array}{c}\text { L. } \\
\text { Naidenoff }\end{array}$ \\
\hline $\begin{array}{l}\% \text { del } \\
\text { tercero/otros }\end{array}$ & 17,68 & 1,26 & 23,9 & 3,39 & 0,23 & 11,37 & 9,4 & 0.61 & 0,81 \\
\hline Partido & Otros & Otros & Otros & Otros & Otros & Otros & Otros & Otros & Otros \\
\hline Candidato & Otros & Otros & Otros & Otros & Otros & Otros & Otros & Otros & Otros \\
\hline
\end{tabular}

Fuente: Elaboración propia a partir de datos de la justicia electoral provincial

\footnotetext{
${ }^{2}$ Los partidos nacionales en la provincia, replican las mismas alianzas que se forman en el nivel nacional. El PJ, desde el 2007 encabeza el Frente para la Victoria, y la UCR desde 2015 encabeza la Alianza UCR Cambiemos.
} 
Tabla 2: Porcentajes de votos obtenidos por el ganador y el segundo candidato más votado, en elecciones para gobernador de Misiones 1983-2015.

\begin{tabular}{|c|c|c|c|c|c|c|c|c|c|}
\hline Elecciones & 1983 & 1987 & 1991 & 1995 & 1999 & 2003 & 2007 & 2011 & 2015 \\
\hline $\begin{array}{l}\text { \% del } \\
\text { Ganador }\end{array}$ & 50 & 47,5 & 48,3 & 53,7 & 53,87 & 47,8 & 38,38 & 69,06 & 63,78 \\
\hline Partido & UCR & PJ & PJ & PJ & PJ & FRCS & FRCS & FRCS & FRCS \\
\hline Candidato & $\begin{array}{c}\text { R. } \\
\text { Barrios } \\
\text { Arrechea }\end{array}$ & $\begin{array}{c}\text { J. } \\
\text { Humada }\end{array}$ & R. Puerta & R. Puerta & J. Rovira & $\begin{array}{c}\text { J. } \\
\text { Rovira }\end{array}$ & $\begin{array}{l}\text { M. } \\
\text { Closs }\end{array}$ & M. Closs & $\begin{array}{c}\text { H. } \\
\text { Passalacqua }\end{array}$ \\
\hline $\begin{array}{l}\text { \% del } \\
\text { segundo }\end{array}$ & 47,4 & 46,5 & 46,8 & 45,8 & 45,68 & 33 & 29,14 & 5,74 & 13,74 \\
\hline Partido & PJ & UCR & UCR & UCR & Al. EJyT & PJ & FpV & $\begin{array}{c}\text { Tr, y Prog. } \\
\text { A }\end{array}$ & FVJ \\
\hline Candidato & $\begin{array}{c}\text { M. } \\
\text { Alterach }\end{array}$ & $\begin{array}{c}\text { M. } \\
\text { Losada }\end{array}$ & $\begin{array}{l}\text { R. Barrios } \\
\text { Arrechea }\end{array}$ & $\begin{array}{l}\text { R. Barrios } \\
\text { Arrechea }\end{array}$ & $\begin{array}{l}\text { R. Barrios } \\
\text { Arrechea }\end{array}$ & $\begin{array}{c}\text { R. } \\
\text { Puerta }\end{array}$ & $\begin{array}{c}\text { P. } \\
\text { Tschirsch }\end{array}$ & $\begin{array}{c}\text { A. } \\
\text { Wipplinger }\end{array}$ & A. Ziegler \\
\hline $\begin{array}{l}\% \text { del } \\
\text { tercero/otros }\end{array}$ & 2,6 & 6 & 4,9 & 0,62 & 0,45 & 19,2 & 32,48 & 25,2 & 22,48 \\
\hline Partido & Otros & Otros & Otros & Otros & Otros & Otros & Otros & Otros & Otros \\
\hline Candidato & Otros & Otros & Otros & Otros & Otros & Otros & Otros & Otros & Otros \\
\hline
\end{tabular}

Fuente: Elaboración propia a partir de datos de la justicia electoral provincial

Tabla 3: Porcentajes de votos obtenidos por el ganador y el segundo candidato más votado, en elecciones para gobernador de Chaco 1983-2015.

\begin{tabular}{|c|c|c|c|c|c|c|c|c|c|}
\hline Elecciones & 1983 & 1987 & 1991 & $1995^{3}$ & 1999 & 2003 & 2007 & 2011 & 2015 \\
\hline$\%$ del Ganador & 47.3 & 50.2 & 37.57 & 50.8 & 63,36 & 53.36 & 46.86 & 66.59 & 52.73 \\
\hline Partido $^{4}$ & PJ & $\mathrm{PJ}$ & ACha & UCR & UCR & UCR & PJ & $\mathrm{PJ}$ & PJ \\
\hline Candidato & $\begin{array}{c}\text { F. } \\
\text { Tenev }\end{array}$ & $\begin{array}{c}\text { D. } \\
\text { Baroni }\end{array}$ & $\begin{array}{c}\mathrm{R} . \\
\text { Tagüinas }\end{array}$ & $\begin{array}{c}\text { A. } \\
\text { Rozas }\end{array}$ & A. Rozas & R. Nikisch & $\begin{array}{c}\text { J. } \\
\text { Capitanich }\end{array}$ & $\begin{array}{c}\text { J. } \\
\text { Capitanich }\end{array}$ & $\begin{array}{c}\text { D. } \\
\text { Peppo }\end{array}$ \\
\hline$\%$ del segundo & 46,20 & 46,50 & 37,22 & 49,2 & 31,21 & 38,48 & 46,61 & 30,23 & 44,15 \\
\hline Partido & UCR & UCR & PJ & $\mathrm{PJ}$ & PJ & PJ & UCR & UCR & UCR \\
\hline Candidato & $\begin{array}{c}\text { L. } \\
\text { León }\end{array}$ & L. León & J. Morales & $\begin{array}{c}\mathrm{F} . \\
\text { Tenev }\end{array}$ & $\begin{array}{c}\mathrm{J} . \\
\text { Capitanich }\end{array}$ & $\begin{array}{c}\mathrm{J} . \\
\text { Capitanich }\end{array}$ & A. Rozas & R. Nikisch & $\begin{array}{c}\text { A. } \\
\text { Ayala } \\
\end{array}$ \\
\hline $\begin{array}{l}\% \text { del } \\
\text { tercero/otros }\end{array}$ & 6,48 & 3,3 & 21,98 & & 5,49 & 8,16 & 6.55 & 3,18 & 3,2 \\
\hline Partido & Otros & Otros & UCR & & Otros & Otros & Otros & Otros & Otros \\
\hline Candidato & Otros & Otros & $\begin{array}{l}\text { Lataza } \\
\text { Lanteri }\end{array}$ & & Otros & Otros & Otros & Otros & Otros \\
\hline
\end{tabular}

Fuente: Elaboración propia a partir de datos de la justicia electoral provincial

Estos resultados electorales, vistos en el largo plazo, dan cuenta de procesos de construcción poder diferenciados para cada provincia. Dichos procesos tienen que ver también, con concepciones de Estado diferenciadas, que le asignan diferentes grados de importancia y significación a la alternancia de personalidades frente al poder político. Estas situaciones terminan dando lugar configuraciones políticas que se visualizan en el plano

\footnotetext{
${ }^{3}$ Los resultados corresponden a la segunda vuelta electoral. En la primera vuelta el Florencio Tenev del Justicialismo obtuvo el $40,83 \%$ de los votos, Ángel Rozas por la UCR obtuvo 32,89\% y Ruiz Palacios por Acción Chaqueña el 18,92\%.

${ }^{4}$ Los partidos nacionales mayoritarios encabezaron alianzas. En el caso de la UCR, entre 1999 y 2011 encabezó el Frente de Todos, y desde el 2015 encabeza la Alianza UCR Cambiemos. En el caso del Justicialismo, en el 2003 encabezó Unión por Nuevo Chaco, el 2007 siguiendo el lineamiento nacional encabezó el Frente para la Victoria, y desde el 2011, encabeza el Frente Chaco Merece Más.
} 
electoral y que son el resultado no sólo de reformas políticas en pos de la perpetuación, sino también de consolidación de liderazgos personalistas al interior de las elites políticas. Todos estos elementos conjugados, afectan al fin y al cabo el desarrollo y la calidad de la democracia, tanto en los planos institucionales como en los resultados electorales. En razón de ello, se presentan diferencias entre las provincias que obedecen a factores propios de cada una de las jurisdicciones, en función de lo cual resulta útil discutir lo que ocurrió en cada una de ellas.

En el caso de Formosa, en concordancia con el planteo de Beck (2012), se trata de un proceso de construcción de hegemonía del Partido Justicialista. Si bien es difícil negar la presencia de relaciones clientelares, la preeminencia del peronismo se desarrolla en un plazo que desde esta óptica de análisis se acerca a la media duración. De esta manera, no se observan cambios de partido de gobierno, pero si se observan cambios en la estructura de la competencia que hasta 1991 se acercaba a ser tripartita, lo cual daba lugar en algunas ocasiones a un bipartidismo imperfecto y en otras a un multipartidismo moderado. Sin embargo la introducción de la posibilidad de reelección y la convivencia de esta regla con la ley de lemas, le permitió al justicialismo enquistarse en el poder ejecutivo local, lo cual trajo como consecuencia la acentuación de una estructura de competencia bipartidista donde en todos los casos el justicialismo se impuso sobre el radicalismo. De esta manera, Gildo Insfran, consolidado y con sus propias mayorías incorpora la reelección indefina en el 2003 y luego para el 2011 deroga parcialmente la ley de lemas, lo que le significa una victoria en la que alcance su máximo caudal electoral el 66,59\% de los sufragios. Este camino a la perpetuación del liderazgo obedeció de esta manera a dos grandes factores, por un lado, las paulatinas modificaciones de la normativa hasta llegar a la reelección indefinida; y por otro lado, las prácticas clientelares y prebendarías en una provincia de frontera y con altos de pobreza. Estos dos grandes factores, favorecieron el desarrollo de una cultura política donde la continuidad del líder resulte indiscutible y la oposición política se desgaste y se debilite de elección.

En la provincia de Misiones, se observa que las distintas reglas de juego político observadas en el periodo en estudio, han permitido el desarrollo de ciclos en lo que los partidos tiendan a perpetuarse en el poder. Así como en la provincia de Formosa han ocupado el cargo tres personas en todo el periodo de tiempo estudiado, en la provincia de Misiones han gobernado tres partidos. De esta manera, como lo señalan Martín y Carlino (2018), estas situaciones obedecen a la influencia de múltiples factores. En esta multiplicidad de factores, es necesario referirse a dos cuestiones claves, por un lado, la 
clase política que busca perpetuarse y a pesar de no contar con reelección indefinida el partido de gobierno logra mantenerse y hasta el momento han estado frente al ejecutivo por cinco periodos consecutivos, y los líderes partidarios una vez cumplidos sus mandatos se convierten en legisladores y en el caso de Rovira, este se encuentra frente a la cámara de representantes desde el año 2007.

Por otro lado, no se logró consenso aun, para adoptar la posibilidad de reelección indefinida para el gobernador, sin embargo los legisladores provincial si cuentan con esa posibilidad por lo cual luego de finalizar los periodos frente al ejecutivo como ya se señaló se postulan para cargos en la cámara de representantes. En función de estos elementos, la perpetuación se desarrolla en dos ámbitos diferentes, por un lado, los partidos frente al ejecutivo; y por otro, los líderes frente a cargos en la cámara de representantes. De esta manera, con los cargos en la cámara de Representantes, los partidos solucionan el problema de la continuidad generado por las limitaciones a la relección que pesan sobre el poder ejecutivo y las elites políticas logran perpetuarse al frente del régimen político provincial. La estructura de la competencia partidaria, si bien permite observar la preeminencia del partido de gobierno, tiende al multipartidismo moderado.

En la provincia del Chaco, las reglas de juego político presentan un mayor grado de estabilidad. En relación con esto, es posible señalar que la ley de lemas no llego a aplicarse en elecciones de ningún tipo o nivel ya que si bien fue adoptada se derogó rápidamente. En cuanto a la incorporación de la reelección, mediante el consenso entre los partidos mayoritarios al momento de la reforma constitucional (MICOZZI, 2001), logro adoptarse la reelección inmediata y abierta para el gobernador. Estas situaciones generaron en la provincia dos efectos marcados sobre el régimen político, por un lado, se acentuó la estructura de la competencia bipartidista; y por otro lado, se observa una alternancia entre los partidos políticos mayoritarios. Estos efectos, permiten señalar que en esta provincia si bien se consolidan los liderazgos, a nivel del poder ejecutivo local no se visibilizan procesos de perpetuación ni de personas ni de partidos.

\section{Conclusiones}

En las provincias en estudio, la incorporación de la posibilidad de tres modalidades de reelección diferentes para el cargo de gobernador, impactó fuertemente sobre los sistemas políticos provinciales. Estas diferencias, tienen que ver con diferentes válvulas que regulan el sistema político, y la presencia de la reelección indefinida para algunos 
cargos pareciera mostrar vestigios que permiten analizar las estructuras de incentivos que ofrece el sistema.

De esta manera, en Formosa donde rige la reelección sin límites, desde 1983 gobernaron la provincia solo tres personas, todas del Partido Justicialista. En Misiones donde la reelecciones están limitadas a sólo una, se dio una alternancia mayor, no obstante el Frente Renovador para Concordia Social, desde su aparición en el sistema de partidos provincial accedió al poder y no ha sido derrotado en las elecciones sucesivas a pesar de la obligada rotación de nombres. Finalmente, en el Chaco donde la reelección permitida es inmediata y abierta, el hecho de poder retornar al poder luego de un periodo legal genera otras dinámicas electorales que dan lugar a una alternancia entre partidos mayoritarios y una rotación mínima de nombres.

Estas diferencias también se reflejan en los sistemas de partidos, ya que en Formosa y Misiones, donde se intentó incorporar la reelección indefinida con diferentes resultados, en largo plazo con el paso del tiempo se vislumbra una tendencia hacia un sistema de partido preponderante, y en el Chaco, donde sólo se llegó a la reelección inmediata y no se dieron nuevos procesos de reforma la tendencia es hacia un bipartidismo que en algunas elecciones toma los matices de imperfección.

En razón de lo señalado, el poder político pareciera estar concentrado en espacios diferentes. En Formosa y con menor claridad en Chaco, el poder político pareciera estar concentrado en torno al gobernador, mientras que en Misiones pudiera pensarse que se circunscribe a la Cámara de Representantes y es ejercido por su presidente quien coincidentemente tiene posibilidades de ser reelecto indefinidamente.

Estas cuestións, invitan a pensar que no sólo se trata de procesos que ubican el poder político en torno a una u otra figura, sino también a maneras de concebir el Estado y su relación con la sociedad civil. Es última situación, se refleja en las reformas electorales y en los modos en los que se desarrollan las mismas, ya que la participación del electorado en las decisiones políticas es diferente y varía de acuerdo con las reglas de juego político propias de cada jurisdicción. De esta manera, se comprueba el supuesto de la hipótesis planteada como válida inicialmente y que tiene que ver con las distintas configuraciones de los sistemas electorales y como una misma regla de juego político tiene diferentes modos de funcionamiento. Las elites políticas, en sentido buscan permanentemente ser favorecidos por las reglas y buscan adaptarlas en tanto pongan en riesgo su continuidad no solo frente al poder sino frente al sistema político. 
En este punto, resulta valido interrogarse, ¿por qué en Chaco no hubo nuevos intentos de reformas en pos de incorporar la reelección indefinida? En el caso de la provincia de Misiones, ¿habrá nuevos intentos de incorporar la reelección indefinida en el futuro, cambiarán nuevamente las reglas electorales, o se mantendrá sin cambios el sistema político? En la provincia de Formosa, ¿se podrá poner límites a la reelección y romper con la hegemonía del Justicialismo? En este sentido, en términos de Sartori (1999, p. 87), es válido expresar que los sistemas de partidos, que tenían características de pluralismos moderados, pasaron a ser bipartidas y con tendencias a consolidar partidos preponderantes.

Finalmente, es necesario manifestar que, la reelección indefinida no permite la oxigenación del poder, dando lugar a modos autoritarios de ejercicio del poder. Los procesos de construcción de hegemonía, no sólo pueden ser observados alrededor de personas y cargos sino también en el control territorial de los diferentes partidos políticos. Las reglas de juego político, y las reformas políticas, parecieran no poder evitar procesos de perpetuación en determinados territorios, y la territorialización del voto conlleva en su interior procesos de territorialización de poder en manos de los partidos políticos.

Los límites a la reelección, favorecen la alternancia, y si bien no impiden la preponderancia de un partido, contribuye al desarrollo de la Democracia. Sin embargo, si se buscara oxigenar el poder al interior de las elites políticas, debiera limitarse la reelección en los cargos de los diferentes poderes, para que un poder no se convierta en una válvula de escape del otro. No obstante, aun modificando las reglas de juego, nuestras sociedades parecieran resultar propensas a que en lo político se desarrollen procesos de construcción hegemonía.

\section{Referencias}

ALMARAZ, María Gabriela. Ambición política por la reelección en las provincias argentinas. Revista de la Sociedad Argentina de Análisis Político, v. 4, n. 2, p. 191- 226, 2010. Disponible en: http://www.scielo.org.ar/scielo.php?script=sci_arttext\&pid=S185319702010000200003. Acceso: 22 feb. 2019.

APTER, David Ernest; ECKSTEIN, Harry; HECKSCHER, Gunnar. Comparative politics: a reader. New York: Free Press, 1963. 
BECK, Hugo Humberto. Gildo Insfrán, gobernador de Formosa. Clientelismo político y hegemonía peronista. En: ENCUENTRO DE GEOHISTORIA REGIONAL, 32., 2012, Resistencia. Actas... Resistencia: Instituto de Investigaciones Geohistóricas - CONICET, Universidad Nacional del Nordeste, 2012. p. 41-50. Disponible en: https://iighi.conicet.gov.ar/wp-content/uploads/sites/29/2017/07/XXXII-EGHRActasDigitales.pdf. Acceso: 23 feb. 2019.

CARDARELLO, Antonio. El irresistible anhelo de inmortalidad. Los gobernadores y la Reelección inmediata en Argentina (1983-2007). Revista de la Sociedad Argentina de Análisis Político, v. 6, n.1, p. 153-194, 2012. Disponible en: https://www.redalyc.org/articulo.oa?id=387136367006. Acceso: 25 marzo 2019.

CORBACHO, Alejandro. Reformas constitucionales y modelos de decisión en la democracia argentina, 1984-1994. Desarrollo Económico, v. 37, n. 148, 591-616, 1998. Disponible en: https://home.udesa.edu.ar/files/humanidades/DT\%201\%20\%20Alejandro\%20Corbacho.pdf. Acceso: 18 marzo 2019.

DE RIZ, Liliana. El debate sobre la reforma electoral en la Argentina. Desarrollo Económico, v. 32, n. 126, p. 163-184, 1992.

DUVERGER, Maurice. Los partidos políticos. México: Fondo de Cultura Económica, 1957.

GARAY, Eugenia. Alternancia de Partidos Políticos en el Gobierno Ejecutivo Subnacional y análisis en particular de la Provincia del Chaco (1983-2011). Tesis (Maestría en Economía Política y Gobierno) - Escuela de Gobierno de la Provincia del Chaco, Universidad Nacional de San Martin, Resistencia, Argentina, 2017.

GARGARELLA, Roberto. El ideal de la democracia deliberativa en el análisis del sistema representativo: algunas notas teóricas y una mirada sobre el caso de la Argentina. Revista Sociedad, n. 6, p. 1-17, 1995.

GERVASONI, Carlos. Democracia y Autoritarismo en las Provincias Argentinas. Revista Aportes (para el Estado y la Administración Gubernamental), v. 15, n. 27, p. 101-116, 2009. Disponible en: http://www.asociacionag.org.ar/pdfaportes/27/10\%20\%20Carlos\%20Gervasoni\%20-

\%20Democracia\%20y\%20autoritarismo\%20en\%20las\%20provincias\%20argentinas.pdf. Acceso: 12 feb. 2019.

JOLÍAS, Lucas; REINA, Augusto. Democracia, patrimonialismo y reelecciones en Argentina: en busca de Conceptos. Revista de Ciencias Sociales, segunda época, v. 3, n.19, p. 177-196, 2011. Disponible en: http://www.unq.edu.ar/advf/documentos/59400abb77261.pdf. Acceso: 18 marzo 2019.

LINZ, Juan. Democracia presidencial o parlamentaria. ¿Qué diferencia implica? En: LINZ Juan; VALENZUELA, Arturo (Comp.). La crisis del presidencialismo: perspectivas comparativas. Madrid: Alianza, 1997. p. 21 - 147.

LIJPHART, Arend. Democracies: Forms, performance, and constitutional engineering. European Journal of Political Research, Wiley-Blackwell, v. 25, n. 1, p. 1-17, 1994. https://doi.org/10.1111/j.1475-6765.1994.tb01198.x 
LIPSET, Seymour; ROKKAN, Stein (Ed.). Party systems and voter alignments: Crossnational perspectives. New York: Free Press, 1967.

MARTÍN, Maria Elena; CARLINO, Milva. 30 años de manipulación de las reglas de juego: La legislación electoral en la Provincia de Misiones 1983-2013. Studia Politicæ, n. 43, p. 65105, 2018. Disponible en: https://dialnet.unirioja.es/servlet/articulo?codigo=6909224. Acceso: 22 marzo 2019.

MICOZZI, Juan Pablo. Reformas institucionales en Chaco, Chubut y La Pampa. Tres procesos convergentes, ¿tres procesos idénticos? En: CONGRESO NACIONAL DE CIENCIA POLÍTICA, 5., 2001, Rio Cuarto. Actas... Río Cuarto: Sociedad Argentina de Análisis Político, 2001. p. 14-17.

MOLINELLI, Guillermo. Los sistemas electorales. En: PINTO, Julio (Comp.) Introducción a la Ciencia Política. Buenos Aires: Eudeba, 1998. p. 351-386.

NOHLEN, Dieter. Sistema de gobierno, sistema electoral y sistema de partidos políticos. México: Tribunal Electoral/IFE/Fundación Friedrich Naumann, 1999.

NOHLEN, Dieter. La reforma de la ley electoral: pautas para una discusión. Reis, n. 16, p. 135-143, 1981. Disponible en: http://www.reis.cis.es/REIS/PDF/REIS_016_09.pdf. Acceso: 12 marzo 2019.

NOHLEN, Dieter. Sistemas electorales y sistemas de partidos. México: Fondo de Cultura Económica, 1994.

ORTIZ DE ROZAS, Victoria. El gran elector provincial en Santiago del Estero (2005-2010): Una perspectiva desde adentro de un "oficialismo invencible". Revista de la Sociedad Argentina de Análisis Político, v. 5, n. 2, p. 359-400, 2011. Disponible en: https://dialnet.unirioja.es/servlet/articulo?codigo=5216234. Acceso: 23 feb. 2019.

PASQUINO, Gianfranco. Sistemas políticos comparados. Buenos Aires: Prometeo, 2004.

PÉREZ LIÑÁN, Alejandro. El método comparativo: fundamentos y desarrollos recientes. Política Comparada, n. 1, p. 1-29, 2008. Disponible en: https://www.politikaperu.org/elmetodo-comparativo-fundamentos-y-desarrollos-recientes.htm. Acceso: 22 feb. 2019.

QUILICI, Federico. El impacto de los diseños institucionales multinivel sobre el sistema de partidos: los casos de las provincias de Misiones y Corrientes. Tesis (Doctorado en Ciencia Política) - Universidad Nacional de San Martin, San Martin, Argentina, 2015.

RATTO, María Celeste. ¿Rechazo a la democracia o al partido de gobierno?: Despejando el camino para el estudio de la accountability electoral o algunas reflexiones sobre la relación entre crisis económica y voto en los últimos 30 años. Revista de la Sociedad Argentina de Análisis Político, v. 7, n. 2, p. 365-377, 2013. https://dialnet.unirioja.es/servlet/articulo?codigo=5205580. Acceso: 25 feb. 2019.

ROKKAN, Stein. Citizens elections parties: approaches to the comparative study of the processes of development. Oslo: Universitetsforlaget, 1970.

SARTORI, Giovanni. Partidos y Sistemas de Partidos. Madrid: Alianza, 1980. 
SARTORI, Giovanni. En defensa de la representación política. Claves de razón práctica, n. 91, p. 2-6, 1999.

SARTORI, Giovanni; MORLINO, Leonardo (Ed.). La comparación en las ciencias sociales. Madrid: Alianza, 1994.

SÁIZ ARNAIZ, Alejandro. Forma de gobierno y estructura del Poder Ejecutivo: el presidencialismo argentino tras la revisión constitucional de 1994. Revista de estudios políticos, n. 97, p. 195-221, 1997. Disponible en: https://dialnet.unirioja.es/servlet/articulo?codigo=27461. Acceso: 12 feb. 2019.

SERRAFERO, Mario. La Reelección Presidencial Indefinida en América Latina. Revista de Instituciones, Ideas y Mercados, n. 54, p. 225-259, 2011. Disponible en: http://www.eseade.edu.ar/files/riim/RIIM_54/54_11_serrafero.pdf. Acceso: 14 marzo 2019.

THIBAUT, Bernard. Presidencialismo, parlamentarismo y el problema de la consolidación democrática en América Latina. Estudios Internacionales, v. 26, n. 102, p. 216-252, 1993. Disponible en: https://revistaei.uchile.cl/index.php/REl/article/view/15406/28685. Acceso: 18 feb. 2019.

TULA, María Inés. La reforma electoral en los' 90: algunos comentarios sobre la Ley de Lemas en la Argentina". En: SIDICARO, Ricardo; MAYER, Jorge; BOTANA, Natalio (Comp.). Política y sociedad en los años del menemismo. Buenos Aires: Ciclo Básico Común, 1995. p. 15 -39.

TULA, María Inés. La reforma política en las provincias argentinas: elementos para su debate. En: INTERNATIONAL CONGRESS OF LATIN AMERICAN SCIENCE ASSOCIATION, 23., 2001, New York. Actas... New York: Latin American Science Association, 2001. p. 06-08. 